\title{
Calculating the electronic transmission properties of semiconducting carbon nanotube Schottky diodes with increase in diameter
}

\author{
Rashid Nizam ${ }^{1}$, S. Mahdi A. Rizvi ${ }^{2}$ and Ameer Azam ${ }^{3}$ \\ ${ }^{1}$ Centre of Excellence in Material Science, Applied Physics AMU, Aligarh, India \\ E-mail: rashid.nizam@gmail.com; phone:0091-9760129889 \\ ${ }^{2}$ Department of Mechanical Engineering, Aligarh Muslim University, Aligarh, India \\ E-mail:mahdirizvi@yahoo.com ${ }^{2}$ \\ ${ }^{3}$ Centre of Nanotechnology, King Abdulaziz, KSA, India \\ E-mail:azam222@rediffmail.com ${ }^{3}$
}

\begin{abstract}
Transmission of twenty-four carbon nanotube geometries to form twelve intramolecular junctions between every two carbon nanotubes have been investigated numerically. The twelve carbon nanotubes are zigzag and rest carbon nanotubes are armchair forming three different kinds of intramolecular junctions named as circumferential defective carbon nanotubes, grouped defective carbon nanotubes and distributed defective carbon nanotubes. Electronic states joining carbon nanotubes form Schottky diode that is analyzed using the tight-binding method. These quantum transmissions through Schottky diodes have been compared among the different defective carbon nanotubes and correlated with the pentagon and heptagon that formed in the intramolecular junction. The transmission coefficient of conduction band always simulated less than the transmission coefficient of valence band in each intramolecular junction irrespective of the joining of carbon nanotubes in the Schottky diodes. The maximum asymmetry of distributed defective carbon nanotubes in transmission is observed more clearly than that for other two defective carbon nanotubes forming Schottky diodes. It is interesting to note that the position of the localized states above and below the Fermi energy level may be controlled with the distribution of the defect pairs and the hexagons around the defects in the defected carbon nanotube.
\end{abstract}

Keywords: carbon nanotube, intramolecular junction, tight-binding method, transmission, Schottky diode.

Manuscript received 12.09.11; revised version received 17.04.12; accepted for publication 14.06.12; published online 25.09.12.

\section{Introduction}

Semiconducting carbon nanotubes have become known as a potential building material for future nanoelectronics. These nanotubes are used to ascertain properties of many ideal devices such as transistors $[1,2]$, diodes [3-5], sensors [6] and non-volatile memory devices $[7,8]$. In recent years semiconducting carbon nanotube Schottky diodes are profitably constructed from asymmetric metallic-semiconducting carbon nanotubes contacts [3-5]. This asymmetric metallicsemiconducting carbon nanotube structure can operate in high frequency applications due to their high carrier mobility [7], small junction areas, high current transport capability [9] as well as low capacitances [10] leading to high cutoff frequency. Two unlike metals at two ends of the semiconducting carbon nanotubes are connected to form this diode. One metal electrode has a lower work function than that of the semiconducting carbon nanotubes in order to make a Schottky contact while the other metal electrode at another end of the semiconducting carbon nanotubes has a higher work function. The other metal electrode is connected with the semiconducting carbon nanotubes to form an ohmic contact. Different groups gave rectification behaviour with the semiconducting carbon nanotubes, but the observed behaviour of the diodes were found to differ from one group to another [3-5]. 
A significant issue for the nature of the contact between the semiconducting carbon nanotubes and the metal electrode is not solved completely [3]. Additional control with understanding is also needed to get better and stabilize the performance of the diodes as well as integrate them into complex circuits. The performance of the semiconducting carbon nanotube Schottky diodes is much dependent on the Schottky barrier height built in the metallic-semiconducting carbon nanotube contacts. This barrier height depends on various material parameters, for example: the metal contact work function, the environment to which the device is exposed, the diameter of the nanotube and the interface structures [11-16]. Constantly advancement of the semiconducting carbon nanotube Schottky diode performance requires a good understanding of the metallic-semiconducting carbon nanotube contacts. Unluckily, this understanding has not reached yet in spite of extensive experimental plus theoretical studies on the topic. The reason that the exact atomic arrangement of the carbon with metal atoms at the interface is barely known during experiments [3-5]. Theoretical studies ignore microscopic description that uses semi-empirical approaches $[17,18]$. Ab initio quantum-mechanical approaches were bounded to only a fraction of the device under equilibrium conditions [15, 19-21]. In this work, we try to investigate the electron transmission properties of the semiconducting carbon nanotube Schottky diodes through similar constructed atomic structures, being based on experimental arrangements by using the tight binding method $[3,4]$.

Single-walled carbon nanotubes (SWNTs) are made of graphite hexagonal carbon-atom lattice into a cylinder. It has drawn a great deal of interests because of their fundamental research importance with tremendous potential technical applications [22]. They may be possibly future molecular electronic devices such as room temperature single electron and field-effect transistors and rectifiers [23-27]. Depending on its helicity and diameter, a SWNT can be either a zigzag or metallic one [28-31]. Thus, two segments of SWNTs with different diameters and helicities can be connected by introducing the pentagon-heptagon defects into the perfect hexagonal network. It will create the metalliczigzag ( $\mathrm{M}-\mathrm{S})$, zigzag-zigzag ( $\mathrm{S}-\mathrm{S})$, or metallicmetallic ( $\mathrm{M}-\mathrm{M})$ intramolecular junctions (IMJs), which may be possible the building blocks for much smaller electronic devices than those with conventional zigzag technology.

Since 1995, electronic properties of the SWNT IMJs have caused a growing number of experimental along with theoretical studies $[32,33]$. It is indicative that a pentagon-heptagon pair with a symmetry axis nonparallel to the tube axis could modify the nanotube helicity by one unit from $(n, m)$ to $(n+1, m-1)$ by Chico et al. [34,35]. Some researchers gave general features of the IMJs in the zigzag configuration [36]. Such kinds of studies recommended that these arrangements could function as nanoscale electronic devices prepared entirely of carbon atoms. Even though great progress in the IMJ research has been made, there are a lot of experimental with theoretical uncertainties about the close relationships between their physical properties as well as corresponding geometrical structures.

In recent times, Min Ouyang et al. calculated the $(21,-2) /(25,-5)$ and $(11,8) /(9,6)$ IMJs by using scanning tunneling microscopy (STE), which gave clear experimental confirmation of the SWNT IMJs [39]. He explained that the $\mathrm{M}-\mathrm{S}$ IMJ has an electronically sharp interface without the localized junction states, while a more extended interface in addition to low-energy localized states are found in the M-M IMJ [39]. They proposed several atomic models to simulate their observed experimental data results.

\section{Model}

To build models of the intramolecular junctions, the defective carbon nanotube DCNT $(n, m)$ indices that correspond to a specific combination of $d$ and $\theta$ [37] that were determined by using an iterative projection matching method [38]. We considered twenty-four carbon nanotube geometries to form twelve intramolecular junctions between every two carbon nanotubes. These carbon nanotubes forming three different kinds of (Stone-Wale defect form) intramolecular junctions, which means that each four carbon nanotubes are identical of either four carbon nanotubes in all respect, expect three different intramolecular junctions between them. The three intramolecular junctions named as circumferential defective carbon nanotubes, grouped defective carbon nanotubes and distributed defective carbon nanotubes as shown in Figs 1, 2 and 3 with the pair of indices $(n, m)$. In the circumferential defective carbon nanotubes, the pentagon and heptagon are formed in the circumference joining two carbon nanotubes as shown in Fig. 1, while in the distributed defective carbon nanotubes these pentagon and heptagon are arranged in the different site on the carbon nanotube as show in Fig. 2. In the grouped defective carbon nanotubes, the pentagon and heptagon grouping is formed on one side of the middle of junction in carbon nanotube as shown in Fig. 3. And in defective carbon nanotubes, the pentagon and heptagon are formed at different sites of the carbon nanotubes.

The configuration of different carbon nanotubes used in the paper with three different intramolecular junctions is as follows from figures, but only pictures of each circumferential DCNT are shown because of a large number of DCNTs. Parameters of the considered configurations are listed in Table.

In some given pair of indices, all three defective carbon nanotubes form the same defective structure that makes the transmission through each defective carbon nanotube exactly the same. It is because the defective carbon nanotubes have the same quantum states for fixed indices which match the experimental data. For the 
semiconducting and armchair portions, various indices are used such as $(5,5)$ and $(7,4)$, respectively. Here each nanotube - $20 \AA$, almost the same radii are used with the total length $40 \AA$. The energy grid of different nanotubes is used \pm 12 and $\pm 2 \mathrm{eV}$ at the lower and upper bounds of a nanotube, respectively. SWNT segments with these indices can be joined faultlessly along a common axis using different configurations of $5 \ldots 7$ defects.

Simulation of carbon nanotube transmission consists of MOSFET like structure that involves a channel made of CNT structure as shown in Fig. 13. It is connected with two electrodes: a source and drain. An insulating thin film, usually silicon dioxide, separates the channel, source, and drain from a third electrode called the gate, as pictured schematically. By applying a voltage $V_{g s}$ between the gate and source, the conductance of the semiconducting channel can be modulated. Charge carriers (electrons or holes) traveling between source and drain encounter a material- and structuredependent energy barrier in the bulk of zigzag. Each time different DCNT is used in the form of channel in the MOSFET structure.

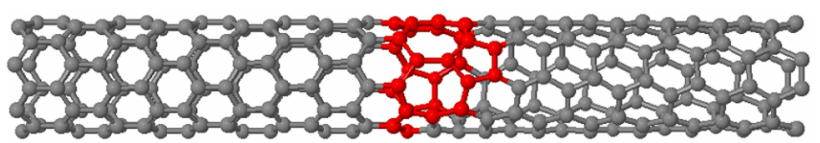

Fig. 1. Intramolecular junction of circumferential DCNT between $7 \times 0$ and $4 \times 4$, respectively.

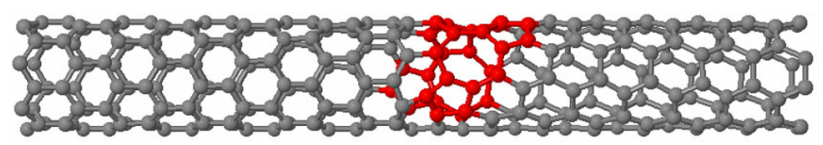

Fig. 2. Intramolecular junction of distributed DCNT between $7 \times 0$ and $4 \times 4$, respectively.

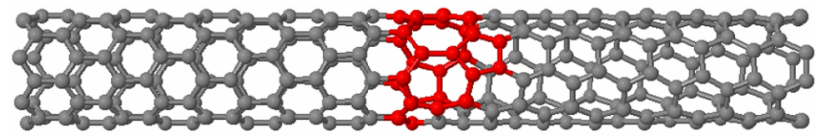

Fig. 3. Intramolecular junction of grouped DCNT between $7 \times 0$ and $4 \times 4$, respectively.

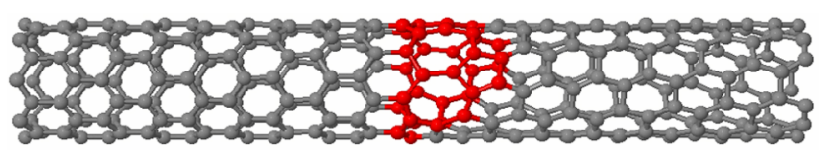

Fig. 4. Intramolecular junction of circumferential DCNT between $8 \times 0$ and $5 \times 5$, respectively.

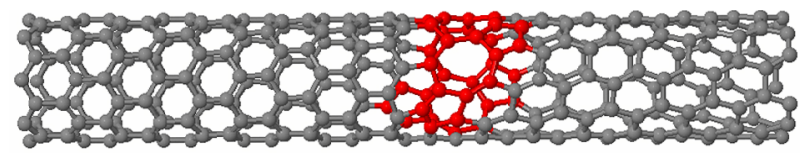

Fig. 5. Intramolecular junction of distributed DCNT between $8 \times 0$ and $5 \times 5$, respectively.

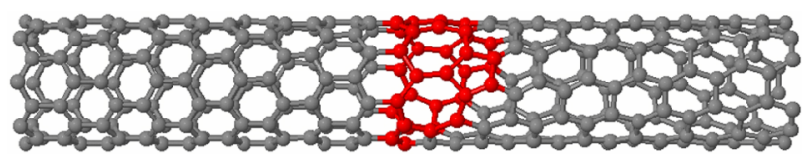

Fig. 6. Intramolecular junction of grouped DCNT between $8 \times 0$ and $5 \times 5$, respectively.

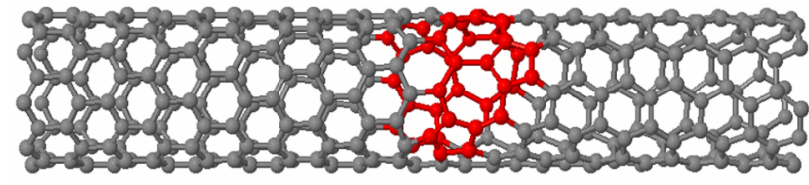

Fig. 7. Intramolecular junction of circumferential DCNT between $9 \times 0$ and $5 \times 5$, respectively.

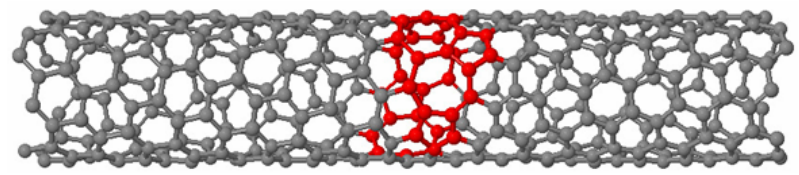

Fig. 8. Intramolecular junction of distributed DCNT between $9 \times 0$ and $5 \times 5$, respectively.

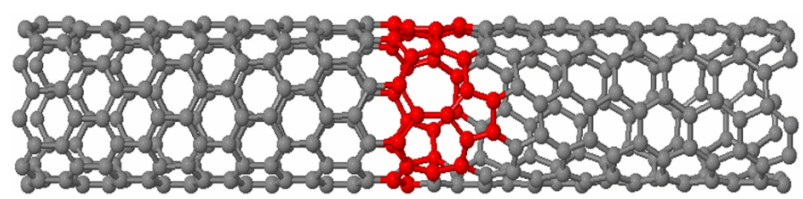

Fig. 9. Intramolecular junction of grouped DCNT between $9 \times 0$ and $5 \times 5$, respectively.

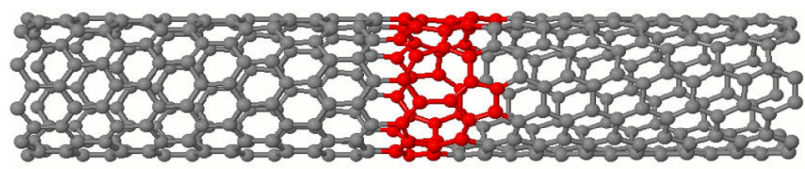

Fig. 10. Intramolecular junction of circumferential DCNT between $10 \times 0$ and $6 \times 6$, respectively.

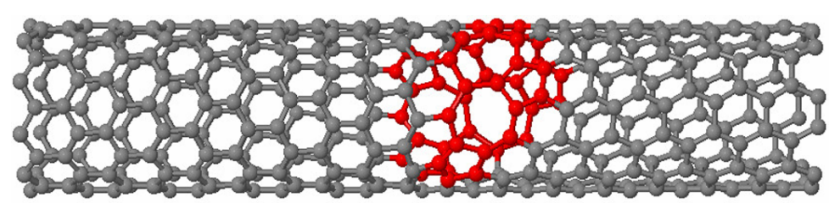

Fig. 11. Intramolecular junction of distributed DCNT between $10 \times 0$ and $6 \times 6$, respectively.

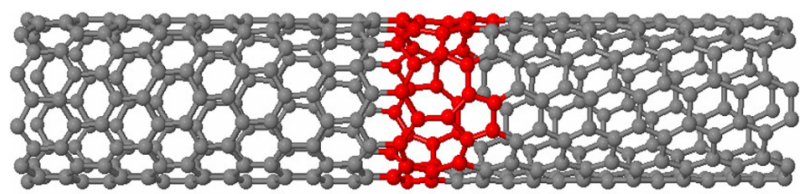

Fig. 12. Intramolecular junction of grouped DCNT between $10 \times 0$ and $6 \times 6$, respectively.

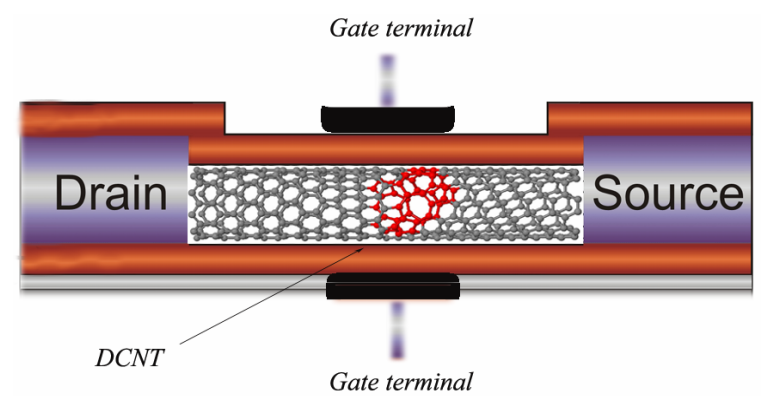

Fig. 13. MOSFET structure involves a channel made of DCNT structure.

(C) 2012, V. Lashkaryov Institute of Semiconductor Physics, National Academy of Sciences of Ukraine 


\section{Simulation details}

The electronic structure of a carbon nanotube (CNT) can be acquired from that of graphene. The wavevector related with the chiral vector $\mathbf{C}_{h}$, in the circumferential direction gets quantized. On the other wavevector associated with the direction of the translation vector $\mathbf{T}$ along the CNT axis remains continuous for an infinite carbon nanotube. These are the boundary conditions of the carbon nanotube.

Various low-energy structural models are shown in these figures, and they have been optimized using molecular mechanics energy minimization. It is possible to evaluate the possibility of these atomic models by calculating the local electronic density of states (DOS) and comparing these with experiment [39]. But very few experiments have done to join the different various carbon nanotubes. Simulation of each intramolecular junction is done, which contains 1000 energy points including on the both grids. The system is described by a simple tight-binding Hamiltonian with a constant nearest neighbour. In this study, the same parameter of hexagon of carbon nanotube is used for the bonds of the pentagon-heptagon pairs of defective carbon nanotube. For a more accurate model, parameters fitted from tightbinding calculations should be used. The Hamiltonian is divided in two blocks corresponding to two semiconducting nanotubes, semiconducting-armchair nanotubes or two armchair nanotubes as shown in various figures. The transmission function can be expressed in terms of the Green functions of the carbon nanotubes and coupling of the carbon nanotubes to the contact electrodes [ $37-39$ ]

$$
T=\operatorname{Tr}\left[\Gamma_{1} G^{r} \Gamma_{2} G^{a}\right] .
$$

Here, $G^{r, a}$ are the retarded and advance Green functions of the carbon nanotube, and $\Gamma_{1,2}$ are functions that describe the coupling of the carbon nanotube to the electrodes. First, let us calculate the Green function of the carbon nanotube for the whole system

$(\in-H)=I$,

where $\epsilon=E+i \eta$ is arbitrary small, and $I$ is the identity matrix. Here, the Hamiltonian of the system is expressed in a discrete real space matrix representation consisting of the carbon nanotube and the semi-infinite electrodes. The above Green function is divided into sub-matrices that correspond to the individual subsystems

$$
\left(\begin{array}{ccc}
G_{L} & G_{L C} & G_{L C R} \\
G_{C L} & G_{C} & G_{C R} \\
G_{L R C} & G_{R C} & G_{R}
\end{array}\right)=\left(\begin{array}{ccc}
\epsilon-H_{L} & h_{L C} & 0 \\
h_{L C}^{\dagger} & \in-H_{C} & h_{C R} \\
0 & h_{C R}^{\dagger} & \in-H_{R}
\end{array}\right)^{-1} .
$$

Here, the matrix $\left(\in-H_{C}\right)$ represents the finite isolated carbon nanotube, $\left(\in-H_{L, R}\right)$ represents the coupling matrices that will be non-zero only for adjacent points in the carbon nanotube and the electrodes, respectively. Then $G_{C}$ can be easily calculated [38]

$G^{r, a}(E)=\left[\epsilon-H_{s}-\Sigma_{1}^{r, a}(E)-\Sigma_{2}^{r, a}(E)\right]^{-1}$,

where $\Sigma_{1}^{r, a}=h_{L C}^{\dagger} g_{L} h_{L C}$ and $\Sigma_{2}^{r, a}=h_{C R} g_{R} h_{C R}^{\dagger}$ defined as the self-energy terms due to the semi-infinite electrode and $g_{\{L, R\}}=\left(\in-H_{\{L, R\}}\right)^{-1}$ are electrodes' Green's functions. The self-energy terms can be considered as effective Hamiltonians that arise from the coupling of the carbon nanotube with the electrodes. By knowing the Green functions, the coupling functions $\Gamma_{1,2}$ can be easily obtained as [38]

$\Gamma_{1,2}(E)=i\left[\Sigma_{1,2}^{r}(E)-\Sigma_{1,2}^{a}(E)\right]$,

where $\Sigma_{1,2}^{a}(E)$ and $\Sigma_{1,2}^{r}(E)$ are the advanced Hermitian self-energy and the retarded Hermitian self-energy that is the conjugate of the advanced Hermitian self-energy [ $40-43]$.

\section{Results and discussion}

The distinctive band structure of metallic carbon nanotubes that is partially responsible for their excellent current transfer capacity that make different from the electronic band structure of conventional metals. The transmission dependence on energy is an important feature of the electronic band structure of metallicsemiconducting, metallic-metallic carbon nanotubes, which is necessary to understand their current-voltage characteristics. The chosen numbers of carbon atoms are taken large enough to avoid the effects of the dangling $\pi$ bonds at the edges of the junctions. We performed a TB calculation at near each intramolecular junction of defective carbon nanotubes.

The transmission dependence on energy for different intramolecular junction carbon nanotubes are shown in Figs 14, 15, and 16. The band structure shows various sub-bands arising from quantization of the wavevector around the circumference of the carbon nanotube irrespective the nature of carbon nanotube structure. The total transmission at a given electron energy is equal to the electron transmission probability times the number of channels but in case for perfect carbon nanotube the transmission without scattering (i.e. transmission probability equal to 1 ) is simply equal to the number of channels. So, the total transmission shows different curves when a sub-band opens or closes. The magnitude of the change in transmission at these curves represents the sub-band degeneracy of the band structure. For example, the different colour lines are not degenerate sub-bands as shown in figure and each contributes one channel for transmission in case of defective carbon nanotube. 


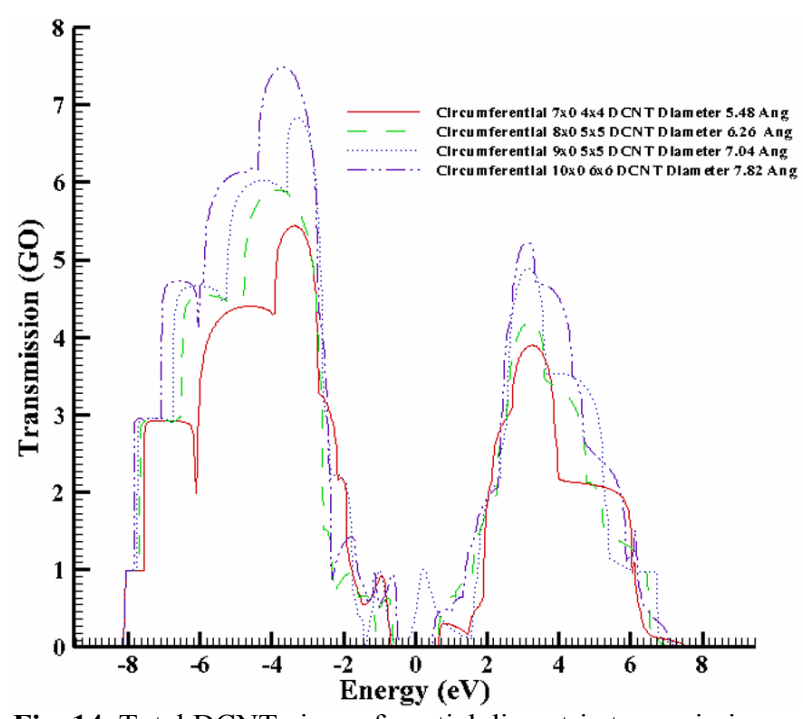

Fig. 14. Total DCNT circumferential diametric transmission.

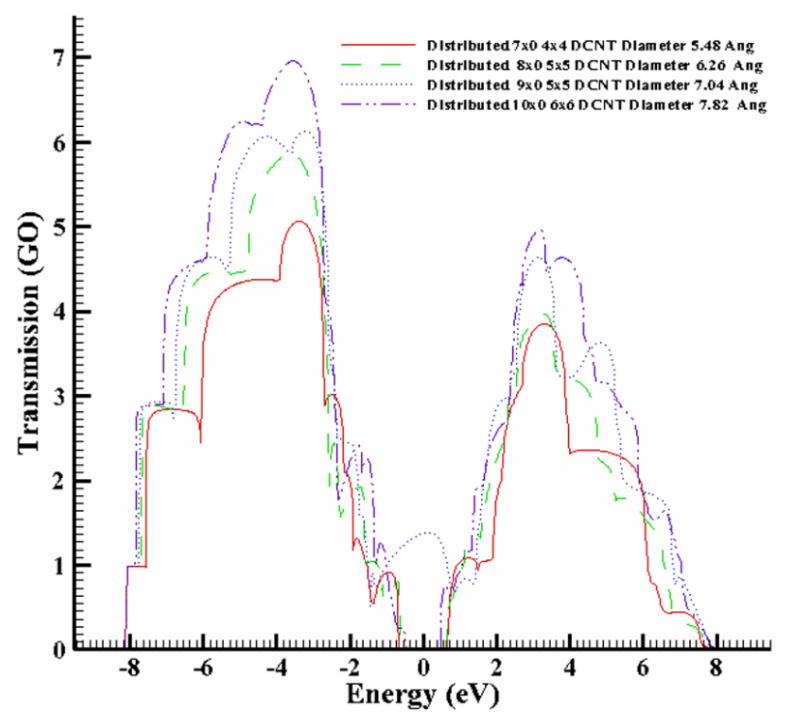

Fig. 15. Total DCNT distributed diametric transmission.

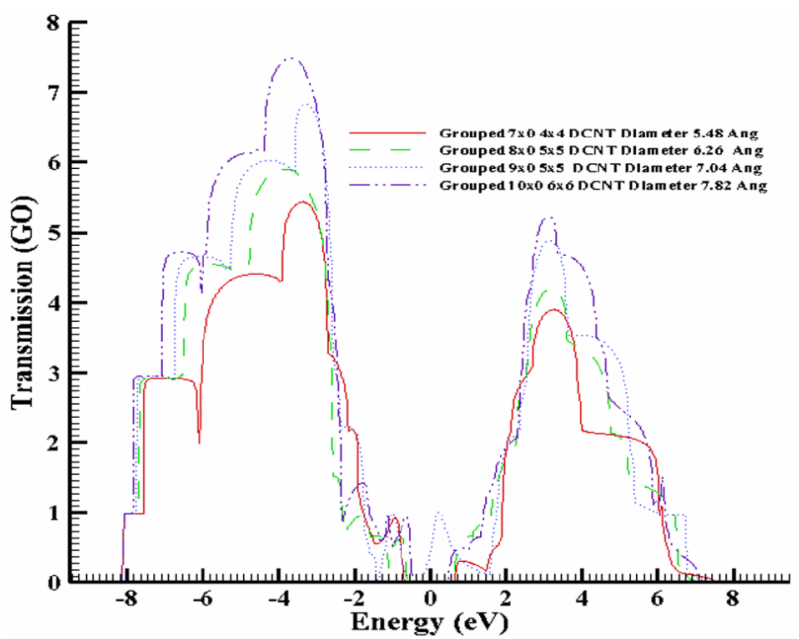

Fig. 16. Total DCNT grouped diametric transmission.
Table. Parameters of the considered configurations.

\begin{tabular}{|c|c|c|c|c|c|c|}
\hline \multirow{2}{*}{ 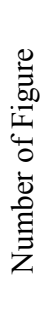 } & \multicolumn{2}{|c|}{ 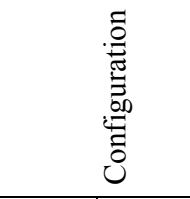 } & \multicolumn{2}{|c|}{ 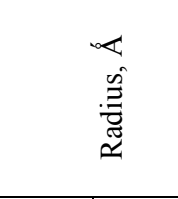 } & \multirow{2}{*}{ 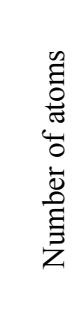 } & \multirow[t]{2}{*}{$\begin{array}{l}\stackrel{0}{0} \\
z\end{array}$} \\
\hline & 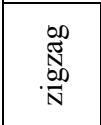 & 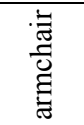 & 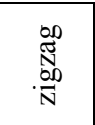 & 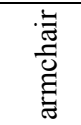 & & \\
\hline 1 & $(7,0)$ & $(4,4)$ & 2.74 & 2.71 & 259 & $\begin{array}{c}\text { in circum- } \\
\text { ferential DCNT }\end{array}$ \\
\hline 2 & $(7,0)$ & $(4,4)$ & 2.74 & 2.71 & 263 & $\begin{array}{c}\text { in distributed } \\
\text { DCNT }\end{array}$ \\
\hline 3 & $(7,0)$ & $(4,4)$ & 2.74 & 2.71 & 259 & $\begin{array}{l}\text { in grouped } \\
\text { DCNT }\end{array}$ \\
\hline 4 & $(8,0)$ & $(5,5)$ & 3.13 & 3.39 & 308 & $\begin{array}{c}\text { in circum- } \\
\text { ferential DCNT }\end{array}$ \\
\hline 5 & $(8,0)$ & $(5,5)$ & 3.13 & 3.39 & 312 & $\begin{array}{c}\text { in distributed } \\
\text { DCNT }\end{array}$ \\
\hline 6 & $(8,0)$ & $(5,5)$ & 3.13 & 3.39 & 308 & $\begin{array}{l}\text { in grouped } \\
\text { DCNT }\end{array}$ \\
\hline 7 & $(9,0)$ & $(5,5)$ & 3.52 & 3.39 & 329 & $\begin{array}{c}\text { in circum- } \\
\text { ferential DCNT }\end{array}$ \\
\hline 8 & $(9,0)$ & $(5,5)$ & $3.52 \AA$ & 3.39 & 335 & $\begin{array}{c}\text { in distributed } \\
\text { DCNT }\end{array}$ \\
\hline 9 & $(9,0)$ & $(5,5)$ & 3.52 & 3.39 & 329 & $\begin{array}{c}\text { in grouped } \\
\text { DCNT }\end{array}$ \\
\hline 10 & $(10,0)$ & $(6,6)$ & 3.91 & 4.07 & 378 & $\begin{array}{c}\text { in circum- } \\
\text { ferential DCNT }\end{array}$ \\
\hline 11 & $(10,0)$ & $(6,6)$ & 3.52 & 3.39 & 384 & $\begin{array}{c}\text { in distributed } \\
\text { DCNT }\end{array}$ \\
\hline 12 & $(10,0)$ & $(6,6)$ & 3.91 & 4.07 & 378 & $\begin{array}{l}\text { in grouped } \\
\text { DCNT }\end{array}$ \\
\hline
\end{tabular}

Some important features that come into light after using these simulations about the defective carbon nanotube interference of the intramolecular junctions forming the carbon Schottky diode configurations are as follows.

1. The position of the localized states above and below the Fermi energy level may be controlled by distribution of the defect pairs (pentagonsheptagons) and the hexagons around the defects in the defected carbon nanotube.

2. The lesser the hexagons around the intramolecular junction defects, asymmetric transmission functions reduce in the carbon Schottky diode, and the transmission becomes step wise.

3. Because of the complex correlation involving the defect structures and the electronic properties, other factors such as the bond lengths of topological defects may be also taken into account. The occupied valence states of the IMJ seem to be mainly affected by the presence of the shared bond between pentagon-heptagons in each carbon Schottky diodes. In each case of carbon Schottky diodes, the greater is the number of pentagonheptagons found in the defective carbon nanotube configuration, the greater variation is observed in 
occupied valences states irrespective of the defective carbon nanotube. However, the number of the defective states below Fermi level does not equal the number of the shared bonds between the pentagon-heptagons.

4. Each pair of zigzag-armchair carbon nanotubes (semiconducting-metallic) or zigzag-armchair carbon nanotubes (metallic-metallic) transmission affect due to the presence of pentagons-heptagons. For a $(n, 0)$ zigzag carbon nanotube, $n-0$ is exactly divisible by 3 , then the carbon nanotube is metallic whereas carbon nanotubes with residuals 1 and 2 of the division $n-m$ by 3 are semiconducting. It is observed that the less fluctuations in the circumferential type defective carbon nanotubes than in the other two kinds, among all types of carbon Schottky diodes.

5. The simulated transmission coefficient of conduction band is always less than the transmission coefficient of the valence band in each intramolecular junction, irrespective of the joining of carbon nanotubes.

6. The asymmetry of the transmission function could be caused by geometries of the semiconductingarmchair carbon nanotube (semiconductingmetallic) interface junction. Besides, the nature of the metallic carbon material and the semiconducting carbon nanotubes has a significant impact on the electron conductance [22]. Figs 14, 15 and 16 shows the transmission functions of all three different semiconducting-armchair carbon Schottky diode configurations under the biases \pm 12 and $\pm 2 \mathrm{eV}$ at the lower and upper bounds of carbon nanotube, respectively. From the figure, the very low energy gap is caused by metal-induced gap states due to the tight coupling between (metallicmetallic) zigzag carbon nanotubes with armchair carbon nanotube atoms. The transmission coefficients in the conduction bands are larger than those in the valence band for all three different models. However, the ratios of the transmission coefficients in the conduction bands to those in the valence bands for these three models are different. Thus, the transmission coefficient ratio which is proportional to the diode rectification ratio is inversely proportional to the strength of the coupling between the semiconducting carbon nanotubes and the armchair (metallic) carbon nanotube atom respective electrodes.

7. The maximum asymmetry of distributed defective carbon nanotubes in transmission is observed as compared to other two defective carbon nanotubes forming Schottky diodes.

8. Some joining the carbon nanotubes having the same quantum configuration structure irrespective of the defect is followed by three degeneracy states, and thus allows to obtain the same transmission for all the defective carbon nanotubes as in Figs 14 and
16. While in other cases, one of degeneracy state gets separated out from the other degeneracy states as shown in Figs 14 and 15. Thus there follow two different transmissions for a given pair of defective carbon nanotube indices. One transmission obtained from one state is different for the other same two given degeneracy states of defective carbon nanotube degeneracy states.

9. It should be noted that in each cases the transmission of carbon Schottky diodes and two metallic-metallic carbon nanotubes (zigzagarmchair carbon one) increase with increase in diameter of the carbon nanotubes irrespective of the defects as shown in Figs 14, 15 and 16. The total number of sub-bands increases with increasing the nanotube diameter, since the amount of quantum numbers arising from quantization of the electron wave function around the nanotube circumference becomes larger, too.

10. It is interesting to note that when two zigzagarmchair carbon nanotubes (semiconductingmetallic) are joined by different defects, the transmission is reduced in each defective carbon nanotube and even in the circumferential as well as grouped defective carbon nanotube cases, it becomes zero as shown in Figs 14 and 16. This is because of the pentagon-heptagons are more effective for this particular quantum configuration structure. While in other cases, all defective carbon nanotubes affect the transmission, but circumferential defective carbon nanotubes least affects and the grouped defective carbon nanotubes have the most influence for this type of quantum configuration structure.

It is observed that the conductance and valence bands have different shapes of transmission across Fermi energy level, where the sub-bands are denoted by the lines cross and become energy zero in each semiconducting carbon nanotube except of one case with a small carbon nanotube diameter. These sub-bands are called crossing sub-bands. The different shapes of transmission across Fermi energy level lies because defective carbon nanotube having bond between pentagon-heptagons at Fermi level and carbon nanotubes extremely sensitive toward the bonding between the carbon atoms.

Location of the wavevector $k$ for defective carbon nanotube changes with carbon nanotube chirality where the crossing occurs. There are only two sub-bands per spin at the Fermi energy independent of carbon nanotube diameter and chirality. It should be noted that the semiconducting sub-bands are also referred to as noncrossing sub-bands as well as crossing sub-bands depending on the indices $(n, 0)$. For a $(n, 0)$ carbon nanotube, $n-0$ is exactly divisible by 3 then the carbon nanotube is metallic, whereas carbon nanotubes with residuals 1 and 2 of the division $n-m$ by 3 are semiconducting. It is observed that the transmission of 
defective carbon nanotubes remains constant at the Fermi level for given pair of indices in each chiral carbon nanotube. Thus, when two zigzag $(8,0)$ armchair $(5,5)$ are joined, no carbon Schottky diode is formed. Every zigzag carbon nanotube transmission becomes zero at the Fermi energy level in the transmission-energy graph irrespective on the number of carbon nanotube defects, while the transmission of chiral armchair carbon nanotubes may get zero depending on the number of pentagons-heptagons in the defective carbon nanotube. The greater number of pentagonsheptagons in the defective carbon nanotube leads the transmission to zero and affect the conductivity of the carbon nanotube, and vice versa.

\section{Conclusion}

It is observed that the carbon nanotube is very sensitive to the atomic structure of their atoms in the carbon nanotubes, and the position of localized states above and below the Fermi energy level may be controlled with the distribution of the defect pairs (pentagons-heptagons) and the hexagons around the defects in the defective carbon nanotube. As the configuration of carbon nanotubes contains the more hexagons around the defects, the less curved are obtained in the transmission and it becomes step-wise. As the configuration of defective carbon nanotubes involves the complex correlation, the bond lengths of topological defects are taken into account. Thus, each pair of zigzag carbon nanotubes - armchair carbon nanotubes forming Schottky diode's transmission affect due to presence of pentagons-heptagons. The simulated transmission coefficient of conduction band is always less than the transmission coefficient of valence band in each intramolecular junction, irrespective of joining the carbon nanotube. It is further observed that some joining of the carbon nanotubes having the same quantum configuration structure irrespective of the defect following the three degeneracy states and thus obtain the same transmission for all the defective carbon nanotubes as in figures. On the other hand, in some cases one of degeneracy state get separated out from the other degeneracy states, and thus it follows two different transmissions for a given pair of defective carbon nanotube indices. One transmission obtained from one state is different for the other similar two given degeneracy states of defective carbon nanotube degeneracy states, except those having the quantum structural configuration.

\section{References}

1. S.J. Tans, A.R.M. Verschueren and C. Dekker // Nature, 393, p. 49 (1998).

2. R.T. Weitz, U. Zschieschang, F. Effenberger, H. Klauk, M. Burghard, and K. Kern // Nano Lett. 7, p. 22 (2007).
3. C. Lu, L. An, Q. Fu, J. Liu, H. Zhang, and J. Murduck // Appl. Phys. Lett. 88, 133501 (2006).

4. M.H. Yang, K.B.K. Teo, W.I. Milne and D.G. Hasko // Appl. Phys. Lett. 87, 253116 (2005).

5. H.M. Manohara, E.W. Wong, E. Schlecht, B.D. Hunt and P.H. Siegel // Nano Lett. 5, p. 1469 (2005).

6. S. Meng, P. Maragakis, C. Papaloukas and E. Kaxiras // Nano Lett. 7, p. 45 (2007).

7. T. Durkop, S.A. Getty, E. Cobas and M.S. Fuhrer // Nano Lett. 4, p. 35 (2004).

8. W.K. Jeong and Q. Jiang // Nanotechnology, 18, 095705 (2007).

9. A. Javey, J. Guo, M. Paulsson, Q. Wang, D. Mann, M. Lundstrom and H. Dai // Phys. Rev. Lett. 92, 106804 (2004).

10. M.S. Dresselhaus, G. Dresselhaus and P. Avouris, Carbon Nanotubes: Synthesis, Structure, Properties, and Applications. Springer, Berlin, 2001.

11. S. Moon, S. Lee, W. Song, J.S. Lee, N. Kim, J. Kim and N. Park // Appl. Phys. Lett. 90, 092113 (2007).

12. Y. Nosho, Y. Ohno, S. Kishimito and T. Mizutani // Nanotechnology, 17, p. 3412 (2006).

13. X. Cui, M. Freitag, R. Martel, L. Brus and P. Avouris // Nano Lett. 3, p. 783 (2003).

14. Y.C. Tseng, K. Phoa, D. Carlton and J. Bokor // Nano Lett. 6, p. 1364 (2006).

15. B. Shen and K. Cho // Phys. Rev. B, 70, 233405 (2004).

16. R.T. Tung // Phys. Rev. B, 64, 205310 (2001).

17. Y. Xue and M.A. Ratner // Phys. Rev. B, 70, 205416 (2004).

18. D. Jimenez, X. Cartoixa, E. Miranda, J. Sune, F.A. Chaves and S. Roche // Nanotechnology, 18, 025201 (2007).

19. W. Zhu and E. Kaxiras // Nano Lett. 6, p. 1415 (2006).

20. S. Okada and A. Oshiyama // Phys. Rev. Lett. 95, 206804 (2005).

21. S. Dag, O. Gulseren, S. Ciraci and T. Yildirim // Appl. Phys. Lett. 83, p. 3180 (2003).

22. C. Dekker // Phys. Today, 52, p. 22 (1999).

23. S. Tans, A.R.M. Verschueren, and C. Dekker // Nature (London), 393, p. 49 (1998).

24. R. Martel, T. Schmidt, H.R. Shea, T. Hertel, and Ph. Avouris // Appl. Phys. Lett. 73, p. 2447 (1998).

25. P.G. Collins, A. Zettl, H. Bando, A. Thess, and R.E. Smalley // Science, 278, p. 100 (1997).

26. Z. Yao, H.W.C. Postma, L. Balents, and C. Dekker // Nature (London), 402, p. 273 (1999).

27. M. Fuhrer, J. Nygard, L. Shih, M. Foreo, Y.-G. Yoon, M.S.C. Mazzoni, H.J. Choi, J. Ihm, S.G. Louie, A. Zettl, and P.L. McEuen // Science, 288, p. 494 (2000).

28. J.W. Mintmire, B.I. Dunlap, and C.T. White // Phys. Rev. Lett. 68, p. 631 (1992). 
29. N. Hamada, S.I. Sawada, and A. Oshiyama // Phys. Rev. Lett. 68, p. 1579 (1992).

30. R. Saito, M. Fujita, G. Dresselhaus, and M.S. Dresselhaus // Appl. Phys. Lett. 60, p. 2204 (1992).

31. T.W. Odom, J.L. Huang, P. Kim, and C.M. Lieber // Nature (London), 391, p. 62 (1998).

32. D. Orlikowski, M.B. Nardelli, J. Bernholc, and C. Roland // Phys. Rev. Lett. 83, p. 4132 (1999).

33. M.S. Ferreira, T. Dargam, R.B. Muniz, and A. Latge // Phys. Rev. B, 62, 16040 (2000).

34. L. Chico, V. H. Crespi, L.X. Benedict, S.G. Louie, and M.L. Cohen // Phys. Rev. Lett. 76, p. 971 (1996).

35. L. Chico, L.X. Benedict, S.G. Louie, and M.L. Cohen // Phys. Rev. B, 54, p. 2600 (1996).

36. J.C. Charlier, T.W. Ebbesen, and Ph. Lambin // Phys. Rev. B, 53, 11108 (1996).
37. M.S. Dresselhaus, G. Dresselhaus, P.C. Eklund, Science of Fullerenes and Carbon Nanotubes. Academic Press, San Diego, 1996.

38. T.W. Odom, J.L. Huang, P. Kim, M. Ouyang, C.M. Lieber // J. Mater. Res. 13, p. 2380 (1998).

39. M. Ouyang, J.-L. Huang, C.L. Cheung, and C.M. Lieber // Science, 291, p. 97 (2001).

40. S. Datta, Electronic Transport in Mesoscopic Systems. Cambridge University Press, Cambridge, 1995.

41. F. Guinea, J. Sanchez-Dehesa, F. Flores, Schottky barrier formation. I. Abrupt metal-zigzag junctions // J. Phys. C: Solid State Phys. 16, p. 6499-6512 (1983).

42. M.P. Lopez Sancho, J.M. Lopez Sancho, J.M.L. Sancho, J. Rubio, Highly convergent schemes for the calculation of bulk and surface Green functions // J. Phys. F: Met. Phys. 14, p. 1205-1215 (1985).

43. I. Deretzis and A. La. Magna // Nanotechnology, 17, p. 5063 (2006). 